Check for updates

Cite this: RSC Adv., 2017, 7, 42129

Received 17th June 2017

Accepted 25th August 2017

DOI: $10.1039 / \mathrm{c} 7 \mathrm{ra06764j}$

rsc.li/rsc-advances

\section{Microencapsulation of 1-hexadecanol as a phase change material with reversible thermochromic properties}

\author{
Bozhen Wu, $\uparrow^{a}$ Liming Shi, $\dagger^{b}$ Qi Zhang ${ }^{* c}$ and Wen-Jun Wang (DD *b
}

Herein, a novel approach for generating a microencapsulated reversible thermochromic phase change material (RTPCM) is described. The system described uses a spirolactone derivative color former, phenolic hydroxyl compound color developer, and 1-hexadecanol as the phase change material and cosolvent. The microencapsulation is achieved by complex coacervation of modified gelatin containing vinyl groups and gum arabic. This is followed by the crosslinking reaction between vinyl groups on modified gelatin and a divinyl crosslinker and subsequent crosslinking reaction with glutaraldehyde to improve stability and encapsulation efficiency. The influence of various key parameters on properties of the microcapsules (encapsulation efficiency, morphology, and particle size) are reviewed including the substitution degree of vinyl groups on modified gelatin, the type and amount of divinyl crosslinkers, and the core/shell ratio. Thermal properties and reversible color reliability of the generated microcapsules were found to be stable after 100 thermal heating and cooling cycles. Such microcapsules are well suited for latent heat storage and as an indicator for the states of energy saturation and consumption in phase change material applications directly through color change.

\section{Introduction}

The continuous increase in the level of greenhouse gas emissions and related environmental issues calls for more effective utilization of renewable energy.$^{1-4}$ While the search for new and renewable energy sources has become a primary research area, the development of energy storage devices is now considered as an equally important issue, since it can reduce the imbalance between supply and demand and improve the performance and reliability of energy systems. Over the past decade, thermal energy storage for sensible, latent, and thermochemical heat has attracted great attention. One of the prospective techniques for storing thermal energy is the application of phase change materials (PCMs). ${ }^{5-7}$ Owing to their high capacity for the reversible storage of thermal energy, PCMs have been used in a variety of applications including solar energy storage, building temperature control, waste heat recovery, and fabric thermoregulation. ${ }^{8-14}$

${ }^{a}$ College of Materials Science and Engineering, Zhejiang University of Technology, Hangzhou 310014, China

${ }^{b}$ State Key Lab of Chemical Engineering, College of Chemical and Biological Engineering, Zhejiang University, Hangzhou, 310027, China. E-mail: wenjunwang@ zju.edu.cn; Fax: +86-571-8795-2772; Tel: +86-571-8795-2772

${ }^{c}$ College of Chemical Engineering, Zhejiang University of Technology, Hangzhou 310014, China. E-mail: qizhang@zjut.edu.cn; Fax: +86-571-8832-0053; Tel: +86571-8832-0917

$\dagger$ These two authors contributed equally to this paper.
Various PCMs, organic and inorganic, have been investigated as latent heat storage materials..$^{8,13,15-19}$ Organic PCMs possess superior properties, such as good chemical and thermal stability, low toxicity, and insignificant supercooling and volume change during phase change processes..$^{20,21}$ Fatty alcohols, including 1-dodecanol, 1-tetradecanol, 1-hexadecanol, and 1-octadecanol, are the most common organic PCMs due to their large latent heat and adjustable phase change temperatures between 24 to $60{ }^{\circ} \mathrm{C} .{ }^{14,17,22}$ The potential use of PCMs is limited due to a variety of issues. Solid PCMs cannot be easily delivered like a fluid and provide for slow heat transfer rates in a large-block form, while molten PCMs are difficult to contain due to liquid migration and are prone to contamination from the surrounding environment.

Encapsulation of PCMs with polymeric shells is considered a promising means to enhance the applicability of PCMs. ${ }^{23,24}$ The segregation by polymeric shells inhibits the loss of the liquid due to leakage, enhances heat transfer rates by increasing surface area, and protects the PCMs from contamination. A variety of processes have been developed for encapsulating the PCMs, including chemical methods (e.g., in situ polymerization, ${ }^{25-27}$ interfacial polymerization, ${ }^{28,29}$ (mini-)emulsion polymerization, ${ }^{30}$ and suspension polymerization ${ }^{31}$ ), physical methods (e.g., spray drying ${ }^{32}$ ), and physicochemical methods (e.g., single and complex coacervation ${ }^{33-37}$ ). While most shell materials for PCM micro- and nano-capsules are synthetic polymers, such as melamine-formaldehyde resin, ${ }^{25}$ ureaformaldehyde resin, ${ }^{26,27}$ poly(methyl methacrylate), ${ }^{38}$ natural 
polymers have recently gained interest due to their reasonable cost, low environmental impact, and wide availability. A common example is gelatin and gum arabic. ${ }^{33,36}$ The drawback of its use is a limited encapsulation efficiency (EE) for PCMs during the complex coacervation of approximately $50-70 \%$ and low durability and serious leakage issues due to the formation of less strong shells. Limited improvements have been achieved through the crosslinking with formaldehyde or glutaraldehyde. ${ }^{33-36}$ Moreover, the PCMs can only handle a fixed phase change latent heat, with no more energy could be stored or released when they reached molten or solid state. Many applications require a simple method to directly indicate the states of energy saturation and consumption of the PCMs. A microencapsulated PCM possessing reversible thermochromic property appears to be an ideal solution for the existing challenges.

Herein, we developed an approach for producing a reversible thermochromic PCM (RTPCM) microcapsule that can not only store the latent heat effectively, but also indicate the states of energy storage and consumption directly through color change. RTPCM microcapsules are produced by complex coacervation of modified gelatin containing vinyl groups and gum arabic, followed by primary crosslinking reaction between the vinyl groups of modified gelatin and divinyl crosslinker, ethylene glycol dimethacrylate, divinylbenzene, or $N, N^{\prime}$-methylenebis(acrylamide), and then succeeding crosslinking reaction with glutaraldehyde, to improve the durability of RTPCM microcapsules and EE. It is believed that the complex coacervation for the preparation of RTPCM microcapsules is advantageous for its simplicity, high productivity, and ecofriendly nature. A thorough investigation was carried out to reveal the effects of substitution degree of vinyl groups on gelatin, the type and amount of the divinyl crosslinkers, and the core/shell material ratio on properties of the RTPCM microcapsules in terms of EE, morphology, and particle size and distribution.

\section{Experimental section}

\section{Materials}

Gelatin (Type B, gel strength 110 to 130 bloom) was purchased from Acros. Gum arabic was obtained from Aladdin Reagent. Methacrylic anhydride and 1-[4-(2-hydroxyethoxy)phenyl]-2hydroxy-2-methyl-1-propan-1-one (Irgacure 2959) were purchased from Sigma-Aldrich and used as received. Ethylene glycol dimethacrylate (EGDMA), divinylbenzene (DVB), and 1hexadecanol (HD) were obtained from Sinopharm Chemical Reagent Co. Ltd. EGDMA and DVB were distilled under vacuum prior to use. $N, N^{\prime}$-Methylenebis(acrylamide) (BisAM) was purified by recrystallization in ethanol. 2-Phenylamino-3-methyl-6di- $n$-butylamino-fluoran was obtained from Connect Wilson (Penglai) Chemie Co. Ltd. and 2,2-bis(4-hydroxyphenyl)propane was purchased from Sinopharm Chemical Reagent Co. Ltd, respectively, and used without further purification. Other chemicals were of analytical grade and used as received.

\section{Synthesis of modified gelatin}

Modified gelatin was prepared by acylation reaction between gelatin and methacrylic anhydride following the published procedure. ${ }^{39,40}$ Gelatin (10 g) was dissolved in $90 \mathrm{~g}$ phosphate buffer at $\mathrm{pH}=8$ and $50{ }^{\circ} \mathrm{C}$, then methacrylic anhydride (0.41$0.82 \mathrm{~g}$ ) was added under vigorously stirring. After $2 \mathrm{~h}$, the reaction product was purified through dialysis for $24 \mathrm{~h}$ against deionized water at $40{ }^{\circ} \mathrm{C}$, followed by freeze-drying. The degree of $\mathrm{NH}_{2}$ group substitution (SD) was determined using the method of Habeeb. ${ }^{\mathbf{1 1}}$ The modified gelatin having different SDs were obtained by changing the addition amount of methacrylic anhydride.

\section{Synthesis of RTPCM microcapsules via complex coacervation}

Synthesis routes of RTPCM microcapsules using complex coacervation of modified gelatin and gum arabic are shown in Scheme 1. 2-Phenylamino-3-methyl-6-di- $n$-butylamino-fluoran, 2,2-bis(4-hydroxyphenyl)propane, and 1-hexadecanol were mixed in a mass ratio of $1: 2: 20$, and melted at $60{ }^{\circ} \mathrm{C}$ to form a homogeneous solution as the core material for subsequent steps. Solutions containing $3 \mathrm{wt} \%$ gum arabic and modified gelatin were prepared in deionized water at $60{ }^{\circ} \mathrm{C}$ and adjusted to $\mathrm{pH}=4.0$ using $10 \mathrm{wt} \%$ acetic acid. The gum arabic solution (100 mL), RTPCM (core material, 3-6 g), Tween 20 (0.2 g), and Span 80 (0.1 g) were added into a flask equipped with a mechanical stirrer. The mixture was heated to $60{ }^{\circ} \mathrm{C}$ and stirred at $1000 \mathrm{rpm}$ for $30 \mathrm{~min}$ to generate a stable dispersion and modified gelatin solution $(100 \mathrm{~mL})$ was added in a dropwise mode. After all of the modified gelatin was added, the dispersion was then stirred at $500 \mathrm{rpm}$ for additional $1 \mathrm{~h}$ and then cooled to $4{ }^{\circ} \mathrm{C}$. During the cooling process, modified gelatin/ gum arabic coacervated particles were separated out from the water. Following this phase separation process, the divinyl crosslinker (0.008-0.024 M) and Irgacure 2959 (50 mg) were added. The microcapsule shells were crosslinked via radical copolymerization between the vinyl groups in modified gelatin and divinyl crosslinker under UV irradiation, shown as crosslinking 1 in Scheme 1 . The crosslinking 1 was carried out in a UV exposure system (DTY-2010, Beijing DETIANYOU Technology Development Co. Ltd) at $365 \mathrm{~nm}$ and $15{ }^{\circ} \mathrm{C}$ under nitrogen atmosphere for $2 \mathrm{~h}$. To enhance the shell mechanical strength of microcapsules, second crosslinking reaction, shown as crosslinking 2 in Scheme 1, was introduced by using glutaraldehyde as crosslinker. The mixture was cooled down to $4{ }^{\circ} \mathrm{C}$, its $\mathrm{pH}$ was adjusted to 9.0 using $5 \mathrm{wt} \%$ sodium hydroxide, and $5 \mathrm{wt} \%$ glutaraldehyde $(10 \mathrm{~mL})$ was introduced. The crosslinking 2 was conducted at $4{ }^{\circ} \mathrm{C}$ for $2 \mathrm{~h}$ and then $60^{\circ} \mathrm{C}$ for another $2 \mathrm{~h}$. The microcapsules were isolated by centrifugation and washed with chloroform and deionized water alternately three times. The recipes for each complex coacervation run are shown in Table 1.

\section{Characterization}

The particle sizes of RTPCM microcapsules were determined with a Coulter LS-230 laser particle size analyzer. The morphology of microcapsules was visualized by using a Hitachi TM-1000 scanning electron microscope (SEM). Thermal properties were determined using a TA Instrument Q200 Differential Scanning Calorimetry (DSC). Approximately $10 \mathrm{mg}$ samples were weighted into aluminum pans. The measurements were carried out in the range of -10 to $80{ }^{\circ} \mathrm{C}$ at a heating/cooling rate 


1

\begin{tabular}{|c|c|c|c|c|c|c|}
\hline Run & $\mathrm{SD}^{b}(\%)$ & Divinyl crosslinker & [Divinyl crosslinker] (M) & Core/shell ${ }^{c}$ & $D_{\mathrm{v}}^{d}(\mu \mathrm{m})$ & $\mathrm{EE}(\%)$ \\
\hline 1 & 0 & - & - & $1 / 2$ & 51.8 & 48.6 \\
\hline 2 & 68 & BisAM & 0.008 & $1 / 2$ & 11.1 & 75.5 \\
\hline 3 & 68 & EGDMA & 0.008 & $1 / 2$ & 26.6 & 73.6 \\
\hline 4 & 68 & DVB & 0.008 & $1 / 2$ & 8.8 & 79.8 \\
\hline 5 & 68 & DVB & 0.016 & $1 / 2$ & 7.3 & 86.6 \\
\hline 6 & 68 & DVB & 0.024 & $1 / 2$ & 13.5 & 84.0 \\
\hline 7 & 42 & DVB & 0.016 & $1 / 2$ & 9.2 & 81.5 \\
\hline 8 & 68 & DVB & 0.016 & $1.5 / 2$ & 9.3 & 85.0 \\
\hline 9 & 68 & DVB & 0.016 & $1 / 1$ & 17.5 & 79.6 \\
\hline
\end{tabular}

of $10{ }^{\circ} \mathrm{C} \mathrm{min}{ }^{-1}$ under a constant stream of $\mathrm{N}_{2}$ at $60 \mathrm{~mL} \mathrm{~min}{ }^{-1}$. After the first heating scan reached $80{ }^{\circ} \mathrm{C}$, the sample was held at this temperature for $3 \mathrm{~min}$ to erase any thermal and processing history. The results from the second heating scan were collected for comparison.

EE is defined as the ratio of the mass of RTPCM encapsulated in microcapsules and initially added, which can be estimated from the phase change enthalpies obtained from the DSC thermograms using the following equation, ${ }^{\mathbf{4 2}}$

$$
\mathrm{EE}=\frac{\Delta H_{\mathrm{m}, \mathrm{p}}}{\Delta H_{\mathrm{m}, \mathrm{t}}} \times 100 \%
$$

where $\Delta H_{\mathrm{m}, \mathrm{p}}$ is the melting enthalpy of RTPCM in the microcapsules $\left(\mathrm{J} \mathrm{g}^{-1}\right)$ and $\Delta H_{\mathrm{m}, \mathrm{t}}$ is the estimated melting enthalpy of RTPCM from the receipt $\left(\mathrm{J} \mathrm{g}^{-1}\right)$. To determine the thermal and color reliability of RTPCM microcapsules, thermal cycling tests were conducted according to the experimental procedure in literatures. ${ }^{38,43}$ The microcapsules were heated to $60{ }^{\circ} \mathrm{C}$, above the melting temperature, and then cooled down to $15{ }^{\circ} \mathrm{C}$ from multiple cycles to examine the stability of color appearance, morphologies, particle sizes, and thermal properties.

\section{Results and discussion}

\section{Preparation of RTPCM microcapsules}

It is essential that the RTPCM can effectively store the latent heat, and show a distinct color change from the state of energy 
fully consumed to energy saturation upon heating until it melts completely (and the inverse change upon cooling until it solidifies completely). ${ }^{\mathbf{4 4 5}}$ The temperature triggering color change should be the same as that for the phase change of PCM. As adopted from previous reports on reversible thermochromic systems, three components were selected for the RTPCM used for encapsulation: (1) spirolactone derivative, 2-phenylamino-3methyl-6-di- $n$-butylamino-fluoran, as color former; (2) phenolic hydroxyl compound, 2,2-bis(4-hydroxyphenyl)propane, as color developer; (3) fatty alcohol, 1-hexadecanol, as PCM and cosolvent for electron donating/accepting reactions between the color former and color developer. ${ }^{44,45}$ The RTPCMs were black at $15{ }^{\circ} \mathrm{C}$, and turned into milky white with light pink at $60{ }^{\circ} \mathrm{C}$, as shown in Scheme 2. The black appearance of the spirolactone derivatives was also reported in other papers. ${ }^{46,47}$ The color switched back to black when the RTPCMs were cooled down to $15{ }^{\circ} \mathrm{C}$. The color change was reversible in multiple heatingcooling cycles. The thermochromic mechanism in microencapsulated RTPCM is given in Scheme 2. When the co-solvent 1-hexadecanol is solidified, the solid environment enhances the interaction between the spirolactone color former and acidic developer, leading to ring-opening of spirolactone to generate black color. Heating the RTPCM to melt 1-hexadecanol, both the color former and developer are dissolved in the co-solvent, encouraging transformation to ring-closed, colorless spirolactone. ${ }^{\mathbf{4 4 , 4 5 , 4 8}}$ The RTPCM comprises 2-phenylamino-3methyl-6-di- $n$-butylamino-fluoran, 2,2-bis(4-hydroxyphenyl) propane, and 1-hexadecanol in a mass ratio of $1: 2: 20$.

As a reference run, the RTPCM microcapsules were prepared via complex coacervation using unmodified gelatin and gum arabic as shell materials and glutaraldehyde as the crosslinker. The volume-average particle diameter $\left(D_{\mathrm{v}}\right)$ and EE are summarized in Table 1 . The measured $D_{\mathrm{v}}$ and EE of microcapsules prepared by unmodified gelatin without crosslinking 1 (Run 1) are $51.8 \mu \mathrm{m}$ and $48.6 \%$, respectively. From the SEM images (Fig. 1a) it can be seen that the microcapsules possessed spherical morphology and many flocs are visible. Although crosslinked by glutaraldehyde, the unmodified gelatin and gum arabic coacervates were not stable enough due to the low crosslinking efficiency as described previously. As a result, some coacervates disassembled during heating and agitating, and flocculated. The leakage of RTPCM core materials during the coacervates disassembling process could further result in a lower EE.

A small peak in the range of approximately $0.05-1.5 \mu \mathrm{m}$ was observed in the plot of volume-based particle size distribution, which could also be seen in Runs 2-9. This is believed to result from some of the gelatin/gum arabic coacervates not migrating to the emulsified RTPCM droplet surfaces, but rather nucleated in the phase separation process, producing small solid particles. A similar observation has been reported by other researchers. ${ }^{33}$ As the percentage of small particles was only about 1-3 v\%, which had little influence on the performance of microcapsules, the effects of small particles will not be discussed in the subsequent sections.

By changing the type and concentration of the divinyl crosslinker, the degree of $\mathrm{NH}_{2}$ group substitution in the modified gelatin, and core RTPCM to shell material ratio, Runs 2-9 RTPCM microcapsules were prepared. Their EE values, particle morphology, and particle sizes were investigated and summarized in Table 1.

Effect of divinyl crosslinkers. The crosslinking 1 reaction between modified gelatin and divinyl crosslinker was introduced during the coacervate formation to improve the stability and EE of RTPCM microcapsules. Three kinds of divinyl crosslinkers including BisAM (Run 2), EGDMA (Run 3), and DVB (Run 4) were used for the system with modified gelatin having $68 \% \mathrm{SD}$ and at a fixed core/shell mass ratio of $1 / 2$. When DVB was used, the $D_{\mathrm{v}}$ was around $8.8 \mu \mathrm{m}$ and the EE reached up to $79.8 \%$ with the formation of segregated spherical microcapsules. Since DVB is a lipophilic crosslinker, it could easily diffuse to the modified gelatin/gum arabic coacervates and participate in crosslinking 1 reaction between modified gelatin and DVB to enhance stable shell formation. By using BisAM as a crosslinker, the microcapsules had larger $D_{\mathrm{v}}$ of $11.1 \mu \mathrm{m}$ and broader particle size distribution than those produced with

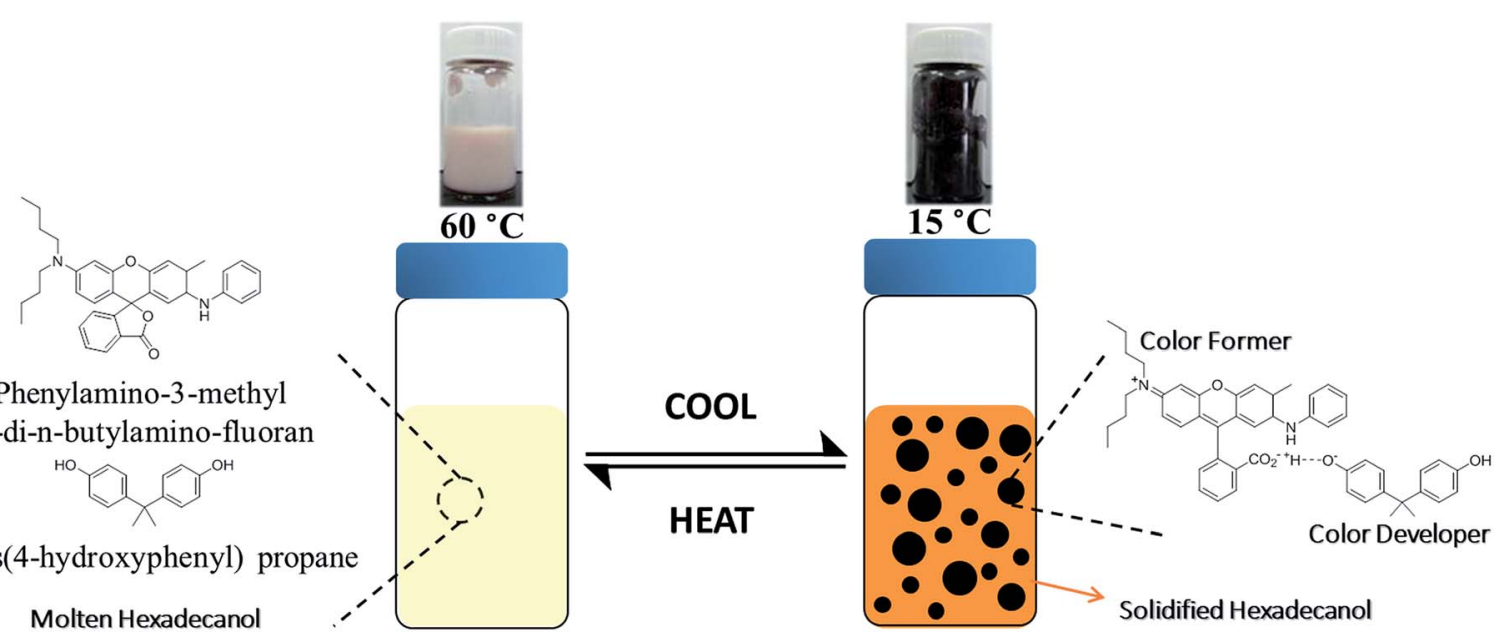

Scheme 2 Thermochromic mechanism in RTPCM. 

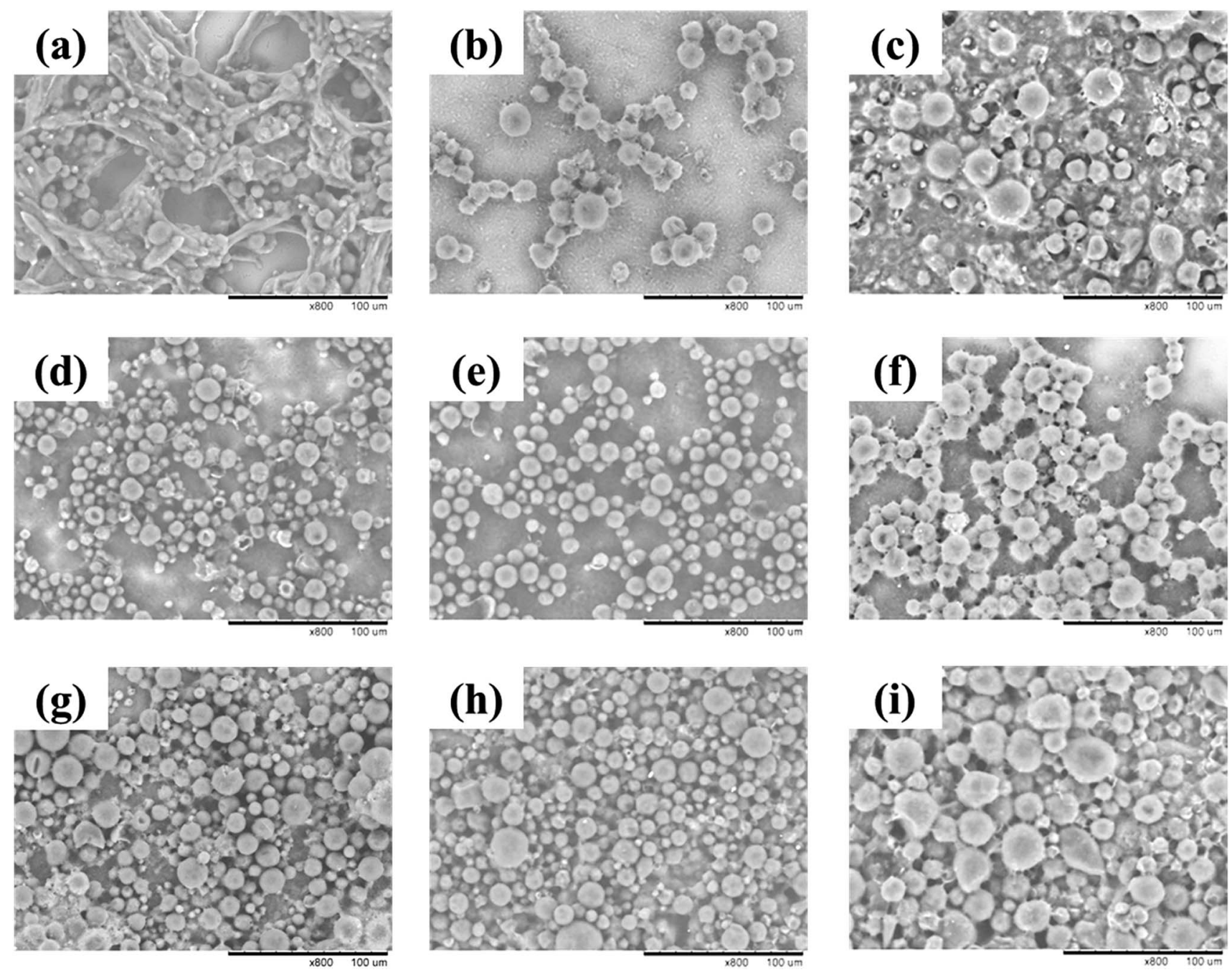

Fig. 1 SEM images of RTPCM microcapsules. (a) Run 1; (b) Run 2; (c) Run 3; (d) Run 4; (e) Run 5; (f) Run 6; (g) Run 7; (h) Run 8; and (i) Run 9. Scale bar: $100 \mu \mathrm{m}$.

DVB. As a more water-soluble crosslinker than DVB, the BisAM dissolved in water could polymerize on their own and react with modified gelatin, thus resulting in particle aggregation from intermolecular crosslinking reaction between the modified gelatin/gum arabic shells, as evident from the multimers in SEM images (Fig. 1b). Severe particle aggregation was observed when EGDMA was used as crosslinker (Fig. 1c). Both intramolecular and intermolecular crosslinking reactions occurred during the formation of microcapsules because of the amphiphilic property of EGDMA, which resulted in particle aggregation and the increase of particle size to $26.6 \mu \mathrm{m}$. Meanwhile, the EE decreased to $73.6 \%$, as compared to the use of DVB in Run 4.

Effect of DVB concentration. Three runs (Run 4-6) using DVB as the divinyl crosslinker were carried out at three different concentrations of $0.008,0.016$, and $0.024 \mathrm{M}$, respectively, and a fixed core/shell mass ratio of $1 / 2$. With the increase of DVB concentrations from $0.008 \mathrm{M}$ to $0.016 \mathrm{M}$, the $D_{\mathrm{v}}$ of microcapsules decreased slightly and increased later. Particle cracking and flocculation occurred in Run 4 (Fig. 1d) due to slightly less stable microcapsule shells. Increasing DVB was beneficial to improve the shell stability of microcapsules due to higher crosslinking density. However, the microcapsules aggregated when the excessive amount of DVB was used (Fig. 1f), which led to the increase in $D_{\mathrm{v}}$. Also, the EE of microcapsules increased to $86.6 \%$ with the increase of DVB concentration to $0.016 \mathrm{M}$, while no distinct change was observed for the subsequent increase of DVB concentration.

Effect of degree of $\mathrm{NH}_{2}$ group substitution on gelatin. Runs were carried out at two different degree of $\mathrm{NH}_{2}$ group substitution on gelatin (SD), Run 5 at $68 \%$ and Run 7 at $42 \%$. With the increase of $\mathrm{SD}$, the microcapsules had a more uniform particle size distribution, smoother morphology (Fig. 1e and $\mathrm{g}$ ), and higher EE. The modified gelatin with higher SD possessed more vinyl groups for crosslinking 1 , which would result in the improvement of crosslinking density in the shell of microcapsules providing the microcapsules with greater stability.

Effect of core/shell mass ratio. Runs 5, 8, and 9 were carried out at core/shell mass ratios of $1 / 2,1.5 / 2$, and $1 / 1$, respectively, to investigate its effect on RTPCM microcapsule formation. The 

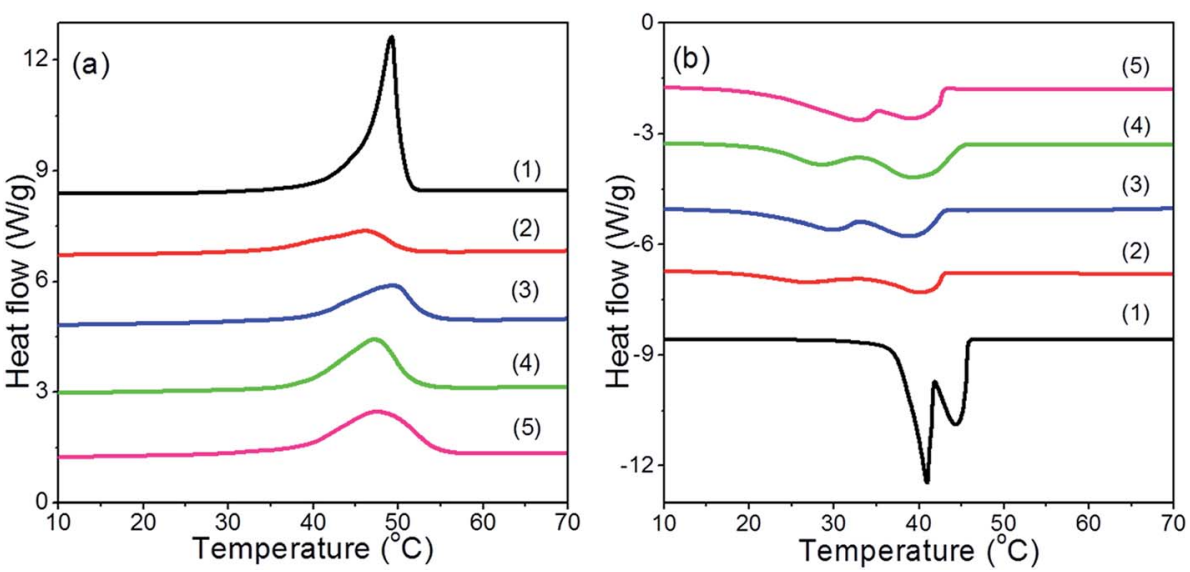

Fig. 2 DSC heating (a) and cooling (b) thermograms of pure (1) and microencapsulated RTPCMs prepared at different core/shell mass ratios: (2) $1 / 2$ with gelatin (Run 1); and (3) 1/2 with modified gelatin (Run 5); (4) 1.5/2 with modified gelatin (Run 8); and (5) 1/1 with modified gelatin (Run 9).

SD of modified gelatin was fixed at $68 \%$, while the DVB concentration was held at $0.016 \mathrm{M}$. With the increase of core/ shell mass ratio, the $D_{\mathrm{v}}$ of microcapsules increased, but the EE decreased. When the core/shell mass ratio increased to 1/1, some irregular spherical microcapsules with larger particle sizes were formed as shown in Fig. 1, which led to a lower EE.

Table 2 Thermal properties of unencapsulated and microencapsulated RTPCMs prepared with different core/shell mass ratios

\begin{tabular}{lccccc}
\hline & & \multicolumn{4}{c}{$T_{\mathrm{c}}\left({ }^{\circ} \mathrm{C}\right)$} \\
\cline { 5 - 6 } Run & $T_{\mathrm{m}}\left({ }^{\circ} \mathrm{C}\right)$ & $\Delta H_{\mathrm{m}}\left(\mathrm{J} \mathrm{g}^{-1}\right)$ & $\alpha$ & $\beta$ & $\Delta H_{\mathrm{c}}\left(\mathrm{J} \mathrm{g}^{-1}\right)$ \\
\hline Pure RTPCM & 49.3 & 204.4 & 43.3 & 41.0 & 207.8 \\
1 & 46.3 & 35.9 & 40.2 & 26.7 & 36.3 \\
5 & 49.2 & 56.5 & 38.6 & 29.9 & 57.8 \\
8 & 47.5 & 72.6 & 39.2 & 28.4 & 72.8 \\
9 & 47.1 & 80.7 & 39.5 & 32.9 & 81.2
\end{tabular}

\section{Thermal properties of RTPCM microcapsules}

Given their intended use, thermal properties of the RTPCM microcapsules are one of their most important aspects. The phase change enthalpy values $(\Delta H)$ strongly depend on the percentage of RTPCM retained in the microcapsules. The thermal properties of pure RTPCM and RTPCM microcapsules having different core/shell mass ratios (Runs 3, 8, and 9) were determined by DSC. The RTPCM microcapsules without crosslinking 1 in the shell (Run 1) were also taken into account for comparison. Their DSC thermograms are shown in Fig. 2.

The melting temperature $\left(T_{\mathrm{m}}\right)$, crystalline temperature $\left(T_{\mathrm{c}}\right)$, melting enthalpy $\left(\Delta H_{\mathrm{m}}\right)$, and crystalline enthalpy $\left(\Delta H_{\mathrm{c}}\right)$ are summarized in Table 2. From the heating thermograms (Fig. 2a), single endothermic peak could be observed for all runs. The $T_{\mathrm{m}}$ of microcapsules in Run 5 using modified gelatin was higher than that of microcapsules in Run 1 with unmodified gelatin, which may be due to different thermal conductivity of the shell materials of microcapsules. The shell materials of microcapsules after crosslinking 1 had better crosslinking

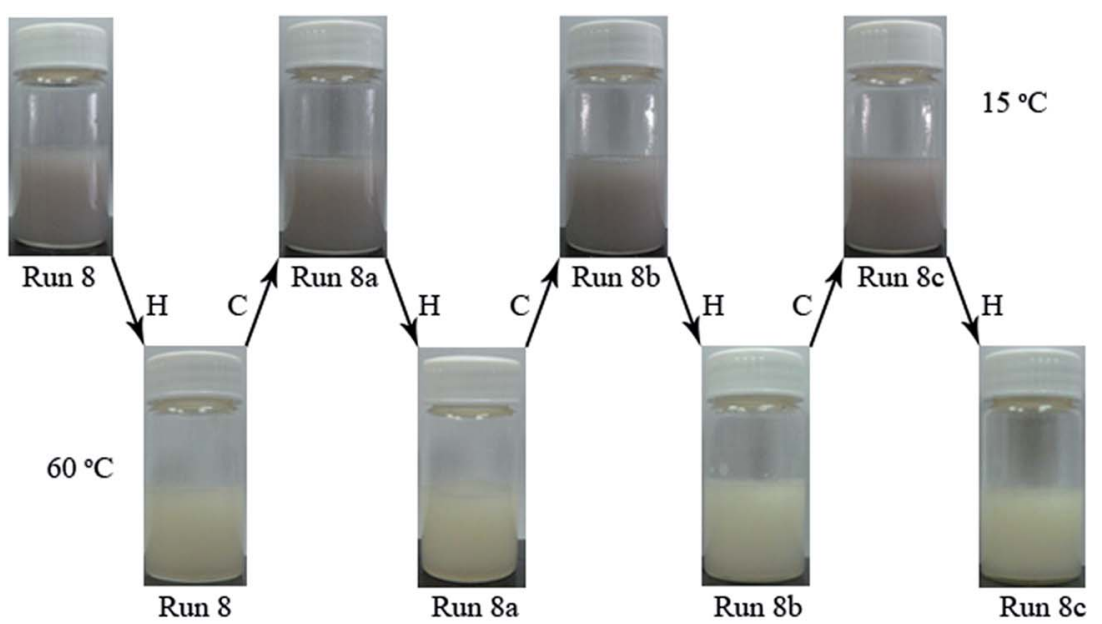

Fig. 3 Color change images of Run 8 RTPCM microcapsules respectively at $15^{\circ} \mathrm{C}$ (gray) and $60{ }^{\circ} \mathrm{C}$ (white) in multiple thermal cycles. $\mathrm{H}$ refers to a heating step while $\mathrm{C}$ is a cooling process in a thermal cycling period. 
Table 3 Characterization of RTPCM microcapsules from Run 8 for multiple thermal cycles

\begin{tabular}{llllll}
\hline Run & Thermal cycles & $D_{\mathrm{v}}(\mu \mathrm{m})$ & $\mathrm{EE}(\%)$ & $T_{\mathrm{m}}\left({ }^{\circ} \mathrm{C}\right)$ & $\Delta H_{\mathrm{m}}\left(\mathrm{J} \mathrm{g}^{-1}\right)$ \\
\hline 8 & - & 9.3 & 85.0 & 47.5 & 72.8 \\
$8 \mathrm{a}$ & 25 & 8.6 & 82.1 & 47.3 & 70.3 \\
$8 \mathrm{~b}$ & 50 & 9.0 & 81.8 & 47.2 & 70.1 \\
$8 \mathrm{c}$ & 100 & 8.9 & 81.1 & 47.7 & 69.5
\end{tabular}

structures but lower thermal conductivities. ${ }^{28,49}$ With the increase of the core to shell mass ratio, $\Delta H_{\mathrm{m}}$ of microcapsules increased from $56.5 \mathrm{~J} \mathrm{~g}^{-1}$ to $80.7 \mathrm{~J} \mathrm{~g}^{-1}$. But the $T_{\mathrm{m}} \mathrm{S}$ in Runs 8 and 9 were lower than that in Run 5, which might be attributed to the higher heat transfer efficiency of the thinner shell and larger particle size. Two exothermic peaks appeared in cooling thermograms for all runs (Fig. 2b), attributing to two exothermic phase transitions of 1-hexadecanol. During the crystallization of 1-hexadecanol, the exothermic phase
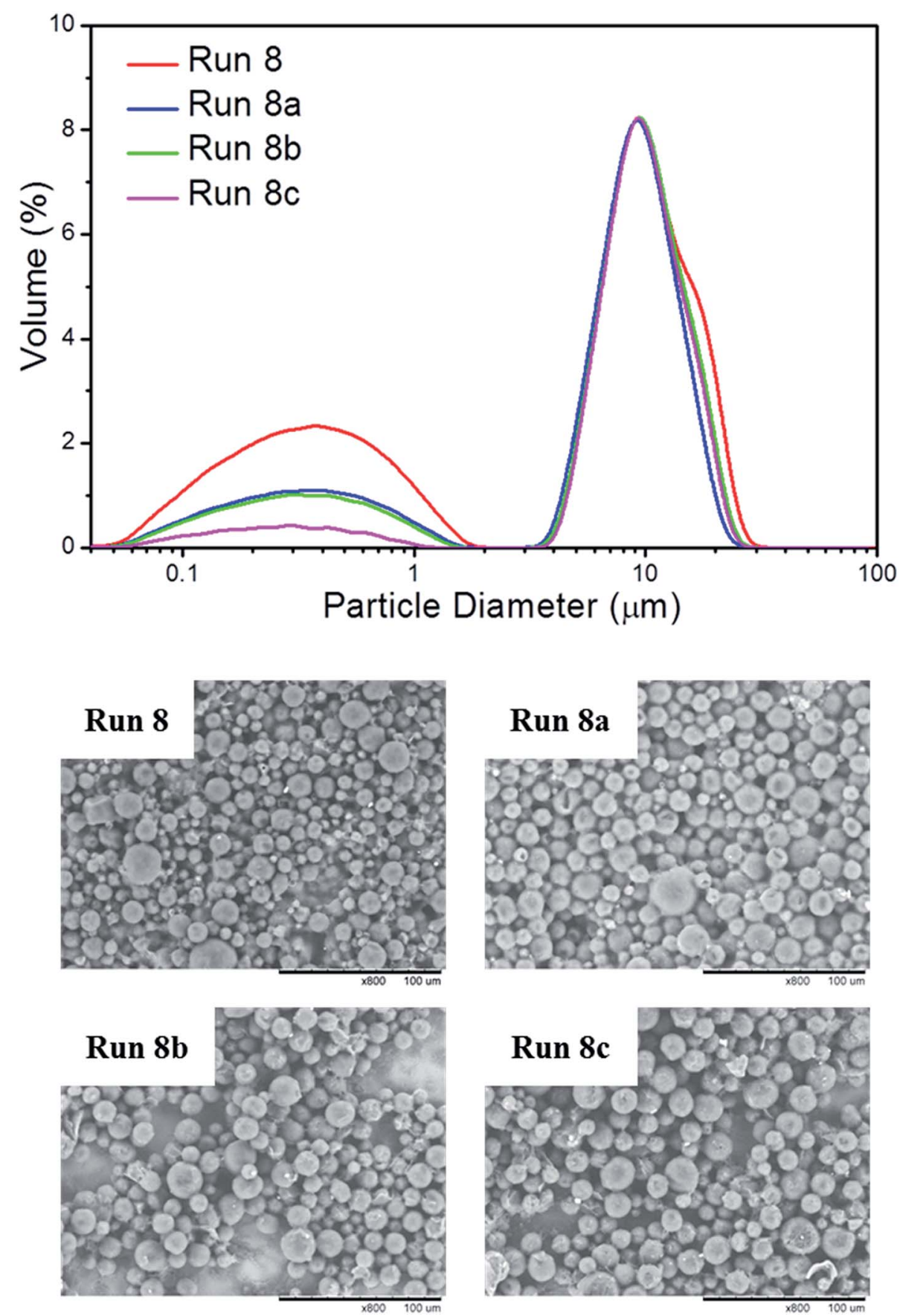

Fig. 4 Volume particle size distributions and SEM images of RTPCM microcapsules from Run 8 after 25 (Run $8 a$ ), 50 (Run 8 b), and 100 (Run 8 c) thermal cycles. Scale bar: $100 \mu \mathrm{m}$. 
transition at higher temperature corresponds to the transformation from a liquid phase to a hexagonally packed solid phase ( $\alpha$-crystal phase), while the exothermic phase transition at lower temperature relates to a transformation from a hexagonally packed solid phase to an orthorhombically packed solid phase ( $\beta$-crystal phase). ${ }^{50,51}$ It was also observed that the $T_{\mathrm{c}}(\alpha)$ of the RTPCM microcapsules was lower than that of pure RTPCM, which was mainly due to heat transfer lag of the crystalline behavior confined inside of microcapsules. Run 5 had the smallest $T_{\mathrm{c}}(\alpha)$ value among the three different core/shell runs owing to the expected lower heat transfer efficiency of the thickest shell.

\section{Thermal and color reliability of RTPCM microcapsules}

To exam the potential application of RTPCM microcapsules in energy storage devices, tests on thermal and color reliability were carried out through thermal cycling. The RTPCM microcapsule sample from Run 8 was used for demonstration as shown in Fig. 3. Obvious color change could be observed in multiple heating-cooling cycles. The dispersion was white at $60{ }^{\circ} \mathrm{C}$, indicating energy saturation, which turned into gray at $15{ }^{\circ} \mathrm{C}$ showing complete energy consumption. The color change was consistent even after 100 thermal cycles. It thus could be concluded that the RTPCM microcapsules had good color reliability.

The $D_{\mathrm{v}}$, EE, and thermal properties of Run 8 after 25, 50, and 100 thermal cycles (Runs 8a, 8b, and 8c, respectively) are summarized in Table 3. The volume particle size distributions and SEM images of RTPCM microcapsules from Run 8 subsequent to various thermal cycles are also shown in Fig. 4. It can be seen that the morphology of microcapsules was preserved after 100 thermal cycles, with a slight decrease in particle size and a $4.5 \%$ EE drop, demonstrating that the RTPCM microcapsules possess good thermal reliability.

\section{Conclusions}

A novel microencapsulated RTPCM with 2-phenylamino-3methyl-6-di- $n$-butylamino-fluoran as color former, 2,2-bis(4hydroxyphenyl)propane as color developer, and 1-hexadecanol as PCM and co-solvent was reviewed. The complex coacervation of modified gelatin and gum arabic, followed by primary crosslinking reaction between vinyl groups of modified gelatin and divinyl crosslinker and subsequent crosslinking with glutaraldehyde were used to produce the RTPCM microcapsules with good stability and high encapsulation efficiency. Thorough study was carried out to reveal the effects of the degree of $\mathrm{NH}_{2}$ group substitution on gelatin, the type and amount of divinyl crosslinkers, and the core/shell ratio on properties of the microcapsules in terms of encapsulation efficiency, morphology, particle size and distribution. The stable microcapsules with latent heat energy $>72 \mathrm{~J} \mathrm{~g}^{-1}, \mathrm{EE}>85 \%$, and uniform particle diameters of approximately 7-10 $\mu \mathrm{m}$ were produced. The properties of the microcapsules could be well maintained after 100 thermal cycles, suggesting a good thermal and color reliability. Such RTPCM microcapsules, which can not only effectively store the latent heat, but also directly indicate the states of energy saturation and consumption by color changing, show promise for use in energy storage devices.

\section{Author contributions}

The manuscript was written with contributions of all authors. All authors have given approval to the final version of the manuscript.

\section{Conflicts of interest}

There are no conflicts to declare.

\section{Acknowledgements}

The authors thank National Natural Science Foundation of China (21420102008 and 21376211) and Chinese State Key Laboratory of Chemical Engineering at Zhejiang University (SKL-ChE-15D03) for financial supports.

\section{References}

1 S. M. Hasnain, Energy Convers. Manage., 1998, 39, 1127.

2 R. Domanski and G. Fellah, Appl. Therm. Eng., 1998, 18, 693.

3 B. Zalba, J. M. Marin, L. F. Cabeza and H. Mehling, Appl. Therm. Eng., 2003, 23, 251.

4 K. Kaygusuz, Energy Sources, 1999, 21, 745.

5 M. Kenisarin and K. Mahkamov, Renewable Sustainable Energy Rev., 2007, 11, 1913.

6 A. M. Khudhair and M. M. Farid, Energy Convers. Manage., 2004, 45, 263.

7 S. Wang, A. L. Cottrill, Y. Kunai, A. R. Toland, P. Liu, W.-J. Wang and M. S. Strano, Phys. Chem. Chem. Phys., 2017, 19, 13172.

8 Q. Yan, C. Liang and L. Zhang, Sol. Energy Mater. Sol. Cells, 2008, 92, 1526.

9 L. F. Cabeza, C. Castellon, M. Nogues, M. Medrano, R. Leppers and O. Zubillaga, Energy Build., 2007, 39, 113.

10 Y. Zhang, S. Wang, Z. Rao and J. Xie, Sol. Energy Mater. Sol. Cells, 2011, 95, 2726.

11 H. H. Ozturk, Energy Convers. Manage., 2005, 46, 1523.

12 T. Nomura, N. Okinaka and T. Akiyama, Resour., Conserv. Recycl., 2010, 54, 1000.

13 A. Sari and K. Kaygusuz, Sol. Energy, 2001, 71, 365.

14 C. Chen, S. Liu, W. Liu, Y. Zhao and Y. Lu, Sol. Energy Mater. Sol. Cells, 2012, 96, 202.

15 J. F. Su, Z. Huang and L. Ren, Colloid Polym. Sci., 2007, 285, 1581.

16 A. Sari, C. Alkan, A. Bicer and A. Karaipekli, Sol. Energy Mater. Sol. Cells, 2011, 95, 3195.

17 C. Chen, Z. Chen, X. Zeng, X. Fang and Z. Zhang, Colloid Polym. Sci., 2012, 290, 307.

18 N. H. S. Tay, M. Belusko and F. Bruno, Appl. Energy, 2012, 90, 288.

19 Z. Tang, A. Liu and Z. Chen, Energy Convers. Manage., 2010, 51, 1459. 
20 M. M. Farid, A. M. Khudhair, S. A. K. Razack and S. Al-Hallaj, Energy Convers. Manage., 2004, 45, 1597.

21 A. Sari and K. Kaygusuz, Renewable Energy, 2001, 24, 303.

22 S. N. Gunasekara, V. Martin and J. N. Chiu, Renewable Sustainable Energy Rev., 2017, 73, 558.

23 X. Wang, J. Niu, Y. Li, Y. Zhang, X. Wang, B. Chen, R. Zeng and Q. Song, AIChE J., 2008, 54, 1110.

24 S. Ma, G. Song, W. Li, P. Fan and G. Tang, Sol. Energy Mater. Sol. Cells, 2010, 94, 1643.

25 Z. Jin, Y. Wang, J. Liu and Z. Yang, Polymer, 2008, 49, 2903.

26 J. Su, L. Ren and L. Wang, Colloid Polym. Sci., 2005, 284, 224.

27 H. Zhang and X. Wang, Colloids Surf., A, 2009, 332, 129.

28 J.-F. Su, L.-X. Wang and L. Ren, Colloids Surf., A, 2007, 299, 268.

29 J.-S. Cho, A. Kwon and C.-G. Cho, Colloid Polym. Sci., 2002, 280, 260.

30 A. Sari, C. Alkan, A. Karaipekli and O. Uzun, Sol. Energy, 2009, 83, 1757.

31 L. Sanchez-Silva, J. F. Rodriguez, A. Romero, A. M. Borreguero, M. Carmona and P. Sanchez, Chem. Eng. J., 2010, 157, 216.

32 A. M. Borreguero, J. L. Valverde, J. F. Rodriguez, A. H. Barber, J. J. Cubillo and M. Carmona, Chem. Eng. J., 2011, 166, 384.

33 L. Bayes-Garcia, L. Ventola, R. Cordobilla, R. Benages, T. Calvet and M. A. Cuevas-Diarte, Sol. Energy Mater. Sol. Cells, 2010, 94, 1235.

34 X.-L. Gu, X. Zhu, X.-Z. Kong and Y. Tan, J. Microencapsulation, 2010, 27, 355.
35 X. Z. Kong, X. Gu, X. Zhu and Z. Zhang, Biomed. Microdevices, 2009, 11, 275.

36 E. Onder, N. Sarier and E. Cimen, Thermochim. Acta, 2008, 467, 63.

37 S. S. Deveci and G. Basal, Colloid Polym. Sci., 2009, 287, 1455.

38 C. Alka, A. Sari, A. Karaipekli and O. Uzun, Sol. Energy Mater. Sol. Cells, 2009, 93, 143.

39 A. I. Van Den Bulcke, B. Bogdanov, N. De Rooze, E. H. Schacht, M. Cornelissen and H. Berghmans, Biomacromolecules, 2000, 1, 31.

40 A. Barbetta, M. Dentini, E. M. Zannoni and M. E. De Stefano, Langmuir, 2005, 21, 12333.

41 A. F. S. A. Habeeb, Anal. Biochem., 1966, 14, 328.

42 R. Yang, Y. Zhang, X. Wang, Y. Zhang and Q. Zhang, Sol. Energy Mater. Sol. Cells, 2009, 93, 1817.

43 A. Sari, Energy Convers. Manage., 2003, 44, 2277.

44 S. M. Burkinshaw, J. Griffiths and A. D. Towns, J. Mater. Chem., 1998, 8, 2677.

45 D. C. MacLaren and M. A. White, J. Mater. Chem., 2003, 13, 1701. 46 T. Ohashi, S. Gontani, K. Miyanaga, T. Kurata, Y. Akatani and S. Matsumoto, Dyes Pigm., 2017, 142, 198.

47 Y. Takahashi, A. Shirai, T. Segawa, T. Takahashi and K. Sakakibara, Bull. Chem. Soc. Jpn., 2002, 75, 2225.

48 D. C. MacLaren and M. A. White, J. Mater. Sci., 2005, 40, 669. 49 H. Zhang and X. Wang, Sol. Energy Mater. Sol. Cells, 2009, 93, 1366.

50 L. Carreto, A. R. Almeida, A. C. Fernandes and W. L. C. Vaz, Biophys. J., 2002, 82, 530.

51 D. W. McClure, J. Chem. Phys., 1968, 49, 1830. 